\title{
Different approaches for objective scoring of experimental post-operative adhesions in the rat model-a description
}

\author{
Bernhard Kraemer • Markus Wallwiener • Nina Petri • \\ Hugh S. Colvin • Sara Brucker • Ralf Rothmund • \\ Taufiek K. Rajab • Christian W. Wallwiener
}

Received: 15 July 2010 /Accepted: 6 October 2010 /Published online: 30 October 2010

(C) Springer-Verlag 2010

\begin{abstract}
The formation of peritoneal adhesions is an issue for surgical research. Different adhesion-inducing models with subsequent scoring systems are descriptively published. The lack of uniformity is a problem as the reproducibility of experimental adhesion research depends strongly on the employed scoring system. A uniform, widely-used score that seeks to describe this relevant surgical condition would be beneficial for inter-surgeon comparability. We present and contrast six scoring systems after the traumatisation of the parietal peritoneum in the rat model. Advantages, disadvantages and statistical considerations of these systems are highlighted. Experimental adhesions were created bilaterally in $n=11$ Wistar rats. This resulted in $n=22$ adhesion areas. Standardised trauma was inflicted to the parietal peritoneum in a linear
\end{abstract}

Taufiek K. Rajab and Christian W. Wallwiener are both joint senior authors

B. Kraemer $(\bowtie) \cdot$ N. Petri $\cdot$ S. Brucker $\cdot$ R. Rothmund

University Hospital for Women, University of Tuebingen,

Calwerstr. 7/1,

72076 Tuebingen, Germany

e-mail: Bernhard.Kraemer@med.uni-tuebingen.de

M. Wallwiener

University Hospital for Women,

Heidelberg, Germany

H. S. Colvin

Addenbrookes Hospital, Cambridge University Hospitals,

Cambridge, UK

T. K. Rajab

Brigham and Women's Hospital, Harvard Medical School,

Boston, MA, USA

C. W. Wallwiener

University Hospital for Women, Technical University of Munich,

Munich, Germany shape $(20 \times 5 \mathrm{~mm})$ using bipolar electrocautery at $40 \mathrm{~W}$. The defect was closed using five interrupted 3/0 Vicryl sutures placed equidistantly. After 14 days, second look laparotomy was performed and number of adhesions, adhesion coverage, adhesion strength, adhesion quality and the attachment to target organs were scored. A total of 43 adhesions formed at the traumatised areas. All of these adhesions could be scored using the six systems described. The correlation coefficient for adhesion coverage as assessed by a measuring stick versus digital computer analysis of the area was 0.649. Every single scoring approach describes different characteristics of adhesions with non-uniform clinical relevance. A multi-dimensional approach that takes account of statistical considerations is inevitable rather than just the use of limited scoring approaches in the experimental setting.

Keywords Peritoneal adhesions · Scoring system · Adhesion induction $\cdot$ Rat model

\section{Introduction}

Peritoneal adhesions can be classified into three categoriescongenital, inflammatory (e.g. post-infectious or due to endometriosis), and post-operative. Post-operative adhesions occur secondary to surgery and they represent the most frequent complication of abdominal and pelvic operations [1]. Consequently, experimental and clinical research has focused on ways to prevent adhesion formation [2,3]. Experiments mostly depend on animal models with objective and reproducible scoring approaches for adhesions. There are a myriad of different adhesion-inducing models with subsequent scoring systems descriptively listed in the literature, both macroscopic and microscopic [4]. These aim to 
describe experiments to improve adhesion barriers $[5,6]$ or optimise surgical instruments with regards to adhesiogenesis. However the lack of uniformity in experimental settings makes the assessment and comparison of adhesion barriers and surgical instruments difficult. As the reproducibility and quality of any adhesion research depends crucially on the employed scoring systems, it is important to consider the strengths and weaknesses of the respective system used and its potential clinical relevance. Regarding adhesions it would be of clinical benefit to have a uniform, widely used score that seeks to describe a relevant surgical condition for inter-surgeon comparability.

Here, we present and contrast six scoring systems we used after the experimental traumatisation of the parietal peritoneum for the comparison of both various induction modalities and different adhesion barriers. We aim to highlight advantages, disadvantages and statistical considerations of these scoring systems to measure features of experimentally induced adhesions in the rat model.

\section{Materials and methods}

\section{Operations}

For the current analysis, experimental adhesions were created bilaterally by an established method $[5,6]$ in $n=$ 11 female, virgin Wistar rats (Crl:WI, Charles River Laboratories, Germany) with a weight range of 220 $280 \mathrm{~g}$. This resulted in $n=22$ scorable adhesion areas. All operations and adhesions were performed by the same surgeons (BK, TR) using powder-free gloves. The experiments were approved by the federal ethical committee (Ethics committee of the University of Tuebingen, \#F 1/06) and performed in accordance with institutional and state guidelines. Anaesthesia was induced with ketamine $(100 \mathrm{mg} / \mathrm{kg})$ and xylazine $(5 \mathrm{mg} / \mathrm{kg})$, intraperitoneally. All operations were performed using aseptic technique and powder-free gloves. After ventral midline incision over a length of $5 \mathrm{~cm}$, standardised trauma was inflicted to the parietal peritoneum on both sides using bipolar electrocautery at $40 \mathrm{~W}$ (Erbe Electrosurgery, Tuebingen, Germany). The traumatised areas were inflicted in a linear shape measuring $20 \times 5 \mathrm{~mm}$ in size, located $1.5 \mathrm{~cm}$ dorsal to the midline and centred at the second pair of mammary glands. The induced peritoneal defect was then closed using five interrupted 3/0 Vicryl sutures (Ethicon, Somerville, USA) placed equidistantly over the defect. The midline incision was closed with continuous 3/0 Vicryl in the musculoperitoneal layer and the intracuticular layer. Post-operatively, the animals received analgesia with buprenorphine $(0.05 \mathrm{mg} / \mathrm{kg})$ subcutaneously three times per day for 2 days and were observed twice daily for signs of complications.

After 14 days, the animals were sacrificed using $\mathrm{CO}_{2}$. Second look laparotomy was performed and all adhesions were scored by an independent surgeon according to the following macroscopic systems:

1. Number of adhesions. In this system the absolute number of individual adhesions formed within the traumatised area is counted [7,8].

2. Adhesion coverage. This may be calculated as the area covered by adhesions divided by the length of the traumatised area $[5,6,9]$. Practically, the area coverage can be quantified in situ manually with a measuring stick (Fig. 1 panel a). Alternatively, digital imaging with a computer programme was used (Corel Photo Paint, Ottowa, Canada). The number of pixels within the area where the adhesion is attached to the peritoneal surface can be counted (Fig. 1 panel b). These two scoring procedures were compared using the correlation coefficient.

3. Adhesion strength. A scoring system based on the difficulty of adhesiolysis may be used (Table 1). For objective accuracy the force required to separate the adhesion from the trauma site can be measured with a digital force metre [10] (Fig. 2).

Fig. 1 Shows measurement of the area covered by adhesions. a Shows the use of a measuring stick in a linear traumatised lesion. b Shows the use of image processing software to select the area where the adhesion is attached to the peritoneal surface
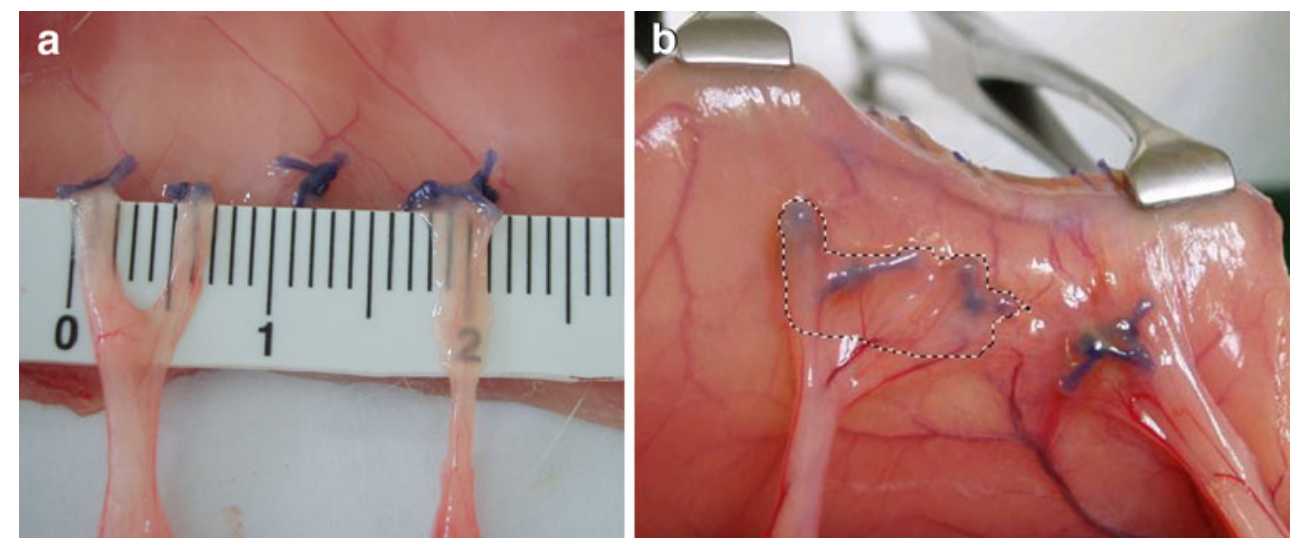
Table 1 Adhesion strength

\begin{tabular}{ll}
\hline Score & Description \\
\hline 0 & No adhesions \\
1 & Adhesions separable by mechanical traction of different force (traction measured by force metre) \\
2 & Sharp dissection required (adhesiolysis by traction causes organ damage) \\
\hline
\end{tabular}

4. Adhesion quality. Scoring of adhesion quality depends on the assessment of morphological criteria (Table 2; Fig. 3).

5. Target organs for the adhesions: omentum, uterine horns, intestine, liver or other organs.

Spearman's rank correlation coefficient analysis (JMP, Version 5.2.1; SAS Institute Inc., Cary, NC) was used.

\section{Results}

A total of 43 adhesions formed to the traumatised areas. All of these adhesions could be scored using the six systems described. The correlation coefficient for adhesion coverage as assessed by a measuring stick versus digital computer analysis of the area was 0.649 (Fig. 4).

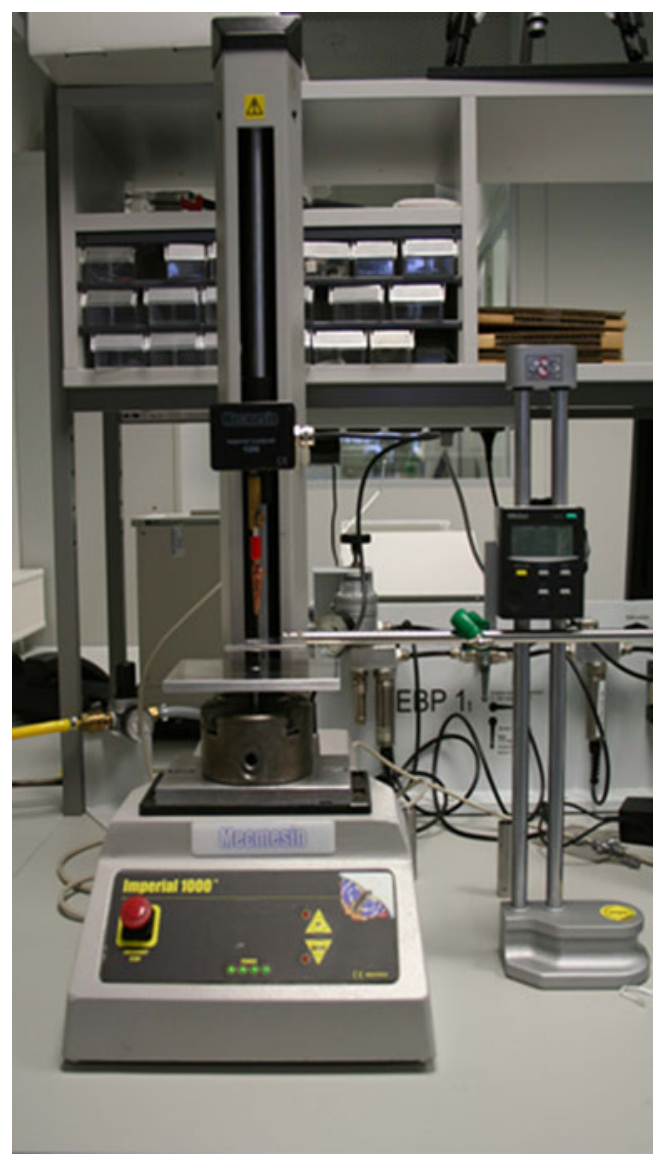

Fig. 2 Shows a digital force metre for measuring the strength of the adhesion

\section{Discussion}

The majority of experiments on adhesiogenesis and prophylaxis are performed on rats as the operation and post-operative care is convenient and less cost intensive than in larger animals such as rabbits and dogs where attempts to score adhesions are also presented and often refer to a trauma of the visceral peritoneum [11] Although interspecies differences in adhesion formation are described in the literature, publications exist that show a similar severity of adhesions after injury to the small bowel of rabbits and the parietal peritoneum of rats [9, 12]. The great number of different experimental models for adhesion induction with subsequent scoring may explain the difficulty to assess and compare prophylactic regimens such as pharmaceuticals, solid or fluid adhesion barriers. Scoring systems have shown to be strongly dependent on the observers with the subsequent subject of interobserver reproducibility [13] As a consequence, an optimal uniform score to describe the extent and severity of adhesions both in the experimental and clinical setting does not yet exist. This article seeks to contrast different measured variables that seem to be most appropriate to characterise experimental adhesions.

Number of adhesions This is a straightforward method that can be achieved quickly and objectively both in situ and ex vivo. However, the absolute number of adhesions is a poor indicator of the total adhesion burden. A single broad adhesive band may cover the same area as several narrow adhesions in the same anatomical region. It is a clinical fact that patients' symptoms do not always correlate with the number of abdominopelvic adhesions $[14,15]$.

Adhesion strength The strength of the adhesion has direct relevance for the assessment of clinical adhesiolysis but not

Table 2 Adhesion quality

\begin{tabular}{ll}
\hline Score & Description \\
\hline 0 & No adhesions \\
1 & Avascular adhesions \\
2 & Filmy vascular adhesions \\
3 & Dense vascular adhesions \\
4 & Organ inclusion \\
\hline
\end{tabular}


Fig. 3 Shows different qualities of adhesions: filmy (indicated by an asterisk), dense (a) and organ inclusion (b)
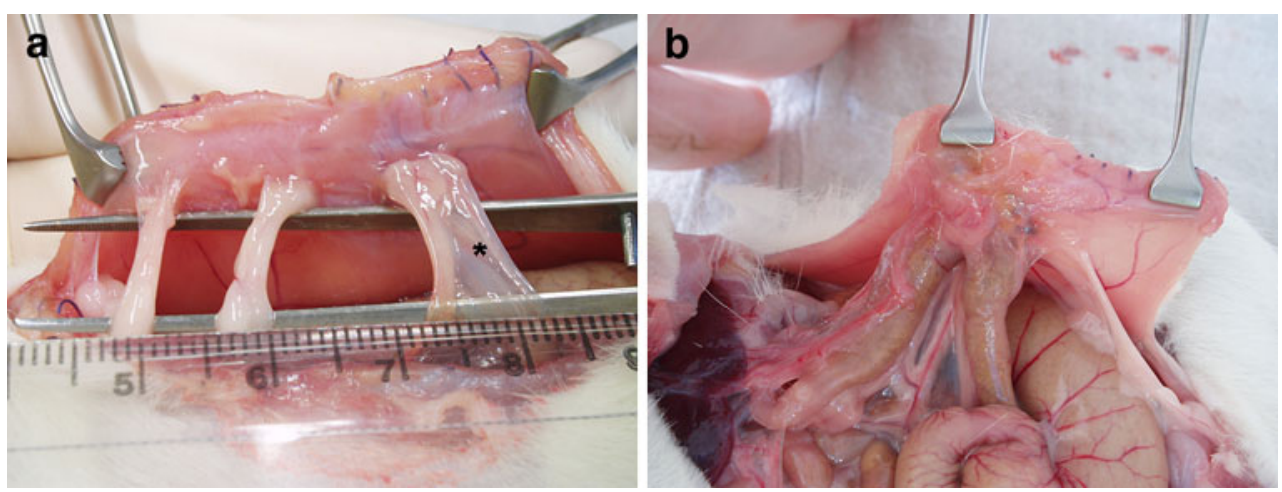

necessarily for the symptoms. The measured variable is continuously distributed. This has implications for the statistical analysis of adhesions that were scored with this system because non-parametric tests such as Student's $t$ test may be employed rather than the more stringent parametric statistical test.

However, in order to accurately and objectively measure the force required to separate adhesions from the peritoneal surface ex-vivo, a force metre is recommended as additional equipment. One problem with this method is where to apply the digital force metre. The strongest point of the adhesion is not necessarily the insertion but could be located at a different point. Moreover, this score cannot be determined in situ because it requires excision of the adhesion. In conclusion, the adhesion strength remains rather difficult to use.

Adhesion coverage This problem can be overcome when the adhesion coverage of the traumatised area is scored. The measured variable is continuously distributed with subsequent advantages for statistical analysis. Here the fraction of the traumatised area covered by adhesions is measured in situ. In linear traumatised lesions, this may be achieved by measuring the length of the area covered with

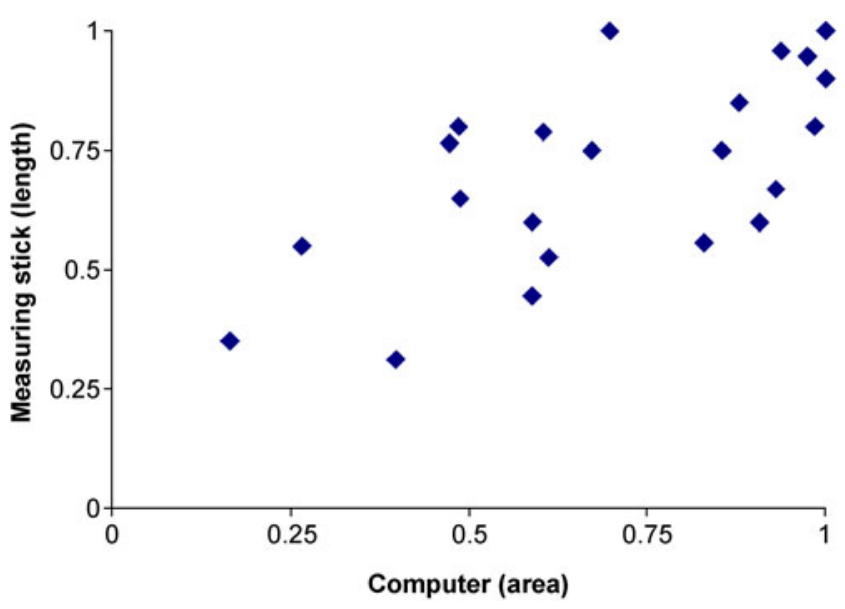

Fig. 4 Shows the correlation for adhesion coverage as assessed by a measuring stick versus digital computer analysis adhesions divided by the length of the traumatised area. Otherwise, digital images may be analysed using a computer programme. However, computer-assisted analysis is time-consuming, and requires calibrated photographic equipment and computer software. Furthermore, our analysis shows that the scores obtained by using the measuring stick (measurement of the attached adhesion length) correlate with the scores derived using the computer (measurement of the attached adhesion area; Fig. 4). Usually, the results of the computer-assisted assessment are obtained after the operation. Thus, this is inconvenient if an in situ assessment is desired. This scoring system can easily be used at the parietal peritoneum but the anatomy of the visceral peritoneum makes this less suitable. The width of the insertion of the adhesion often does not correspond with the width of the more distant adhesive tissue. Therefore measurement of the fraction of the traumatised area covered by adhesions does not accurately reflect the adhesion width further along the adhesion, distant from the insertion. The changes in the abdominopelvic anatomy depend on the fraction of the traumatised area covered by adhesions. As the late complications caused by adhesions are caused by changes in the abdominopelvic anatomy, this is clinically relevant.

Adhesion quality An advantage here is that this score can be quickly used in situ and it is based on morphological terms. However, the adhesion quality does not affect adhesion quantity and the score is observer dependent. The clinical relevance of this score can be considered as high since it can provide technical information for the planned surgical adhesiolysis.

Adhesion targets This score is quick, objective and reproducible. It can be derived in situ and describes anatomical conditions. However, it is based on a nominal scale without a certain rank order. As already mentioned before, from a clinical point of view it remains unclear how adhesions to different targets correlate with possible adverse complications. Again the description of target tissue is a useful technical application for the planning of adhesiolysis. 


\section{Statistical considerations}

Measurement can be classified into different categories of scales [16]. Categorical scoring systems relate to unordered features of peritoneal adhesions. The central tendency of a sample of categorical measurements may only be described using the mode. However, the median and mean cannot be defined. With regards to adhesion scoring systems, this includes classification of the target organs of adhesions (e.g. intestine, pelvic structures, liver). Here, it is not clear whether adhesions to intestinal tissue should be considered to be worse than adhesions to pelvic structures for example.

In contrast, ordinal scoring systems describe features of adhesions that can be assigned a rank order on the basis of a non-linear increase in severity. The central tendency of a sample of ordinal measurements can be statistically described by the mode, median and percentile. However, means or the standard deviation should not be defined. Ordinal scoring systems include the qualitative score assigned in the current paper. Vascular adhesions (score 1) are clearly worse than avascular adhesions (score 2) but it is not clear whether they are exactly twice as bad.

Finally, ratio scales measure the ratio between a magnitude of a continuous quantity of a feature of adhesions and a unit magnitude of the same kind. All statistical measures can be used for a variable measured at the ratio level, as all necessary mathematical operations are defined. The central tendency of a variable measured at the ratio level can be represented by the mode, median, arithmetic mean, or geometric mean with the standard deviation. Ratio-based scoring systems include measurements of the length, area and strength of the adhesions.

\section{Conclusion}

We offer and compare a description of scoring systems that contain measured variables of experimental adhesions. Every single scoring approach describes different characteristics of adhesions with non-uniform clinical relevance. Therefore a multi-dimensional approach that takes account of statistical considerations seems to be inevitable rather than just the use of limited scoring approaches in the experimental setting.

Acknowledgements We gratefully acknowledge the help of veterinarians Dr. Iglauer, Dr. Gerold and Dr. Scheuerlen in the perioperative care of the animals.

Declaration of interest The authors report no conflicts of interest. The authors alone are responsible for the content and writing of the paper.

\section{References}

1. Rajab TK, Wallwiener M, Talukdar S, Kraemer B (2009) Adhesion-related complications are common, but rarely discussed in preoperative consent: a multicenter study. World J Surg 33 (4):748-750

2. De Wilde RL, Trew G (2007) Postoperative abdominal adhesions and their prevention in gynaecological surgery. Expert consensus position. Gynecol Surg 4:161-168

3. De Wilde RL, Trew G (2007) Postoperative abdominal adhesions and their prevention in gynaecological surgery. Expert consensus position. Part 2-steps to reduce adhesions. Gynecol Surg 4:243253

4. Ozel H, Avsar FM, Topaloglu S, Sahin M (2005) Induction and assessment methods used in experimental adhesion studies. Wound Repair Regen 13(4):358-364

5. Rajab TK, Wallwiener CW, Brochhausen C, Hierlemann H, Kraemer B, Wallwiener M (2009) Adhesion prophylaxis using a copolymer with rationally designed material properties. Surgery 145(2):196-201

6. Rajab TK, Wallwiener M, Planck C, Brochhausen C, Kraemer B, Wallwiener CW (2010) A direct comparison of Seprafilm, Adept, Intercoat and Spraygel for adhesion prophylaxis. J Surg Res 161 (2):246-249

7. Reed KL, Fruin AB, Gower AC, Stucchi AF, Leeman SE, Becker JM (2004) A neurokinin 1 receptor antagonist decreases postoperative peritoneal adhesion formation and increases peritoneal fibrinolytic activity. Proc Natl Acad Sci USA 101 (24):9115-9120

8. Reed KL, Fruin AB, Bishop-Bartolomei KK, Gower AC, Nicolaou $\mathrm{M}$, Stucchi AF et al (2002) Neurokinin-1 receptor and substance $P$ messenger RNA levels increase during intraabdominal adhesion formation. J Surg Res 108(1):165-172

9. Holmdahl L, al-Jabreen M, Risberg B (1994) Experimental models for quantitative studies on adhesion formation in rats and rabbits. Eur Surg Res 26(4):248-256

10. Novitsky YW, Harrell AG, Cristiano JA, Paton BL, Norton HJ, Peindl RD et al (2007) Comparative evaluation of adhesion formation, strength of ingrowth, and textile properties of prosthetic meshes after long-term intra-abdominal implantation in a rabbit. J Surg Res 140:6-11

11. Leach RE, Burns JW, Dawe EJ, SmithBarbour MD, Diamond MP (1998) Reduction of postsurgical adhesion formation in the rabbit uterine horn model with use of hyaluronate/carboxymethylcellulose gel. Fertil Steril 69(3):415-418

12. Burns J (1996) A hyaluronate based gel for the prevention of postsurgical adhesions: evaluation in two animal species. Fertil Steril 66:814-821

13. Demirturk F, Aytan H, Caliskan AC (2006) Comparison of the adhesion scoring systems used in animal models and assessment of interobserver reproducibility. Aust N Z J Obstet Gynaecol 46 (4):356-359

14. Stark M, Hoyme U, Stubert B, Kieback D, DiRenzo G (2008) Post-cesarean adhesions - are they a unique entity? J Matern Fetal Neonatal Med 21(8):513-516

15. Alpay Z, Saed G, Diamond M (2008) Post-operative adhesions: from formation to prevention. Semin Reprod Med 26:313-321

16. Stevens SS (1946) On the theory of scales of measurement. Science 103:677-680 